\title{
Sharp constant of Hardy operators corresponding to general positive measures
}

Xudong $\mathrm{Nie}^{1 *}$ (D) and Dunyan Yan²

"Correspondence: nxd2017@outlook.com

'Department of Mathematics and Physics, Shijiazhuang Tiedao University, Shijiazhuang, P.R. China Full list of author information is available at the end of the article

\begin{abstract}
We investigate a new kind of Hardy operator $H_{\mu}$ with respect to arbitrary positive measures $\mu$ and prove that $H_{\mu}$ is bounded on $L^{p}(d \mu)$ with an upper constant $p /(p-1)$. Moreover, we characterize a sufficient condition about the measure which makes $p /(p-1)$ to be the $L^{p}$-norm of $H_{\mu}$.
\end{abstract}

MSC: $42 \mathrm{~B} 20 ; 42 \mathrm{~B} 35$

Keywords: Hardy operator; Best constant; Sharp problem

\section{Introduction}

Let $\mu$ be a positive measure on $[0, \infty)$ and $f$ be a nonnegative $\mu$-measurable function. Define Hardy operator with respect to the measure $\mu$ by

$$
H_{\mu} f(x)=\frac{1}{\mu([0, x])} \int_{[0, x]} f(t) d \mu(t)
$$

if $0<\mu([0, x])<\infty$, and set $H_{\mu} f(x)=0$, if $\mu([0, x])=0$ or $\infty$.

Observe that if $\mu$ is Lebesgue measure, then $H_{\mu}$ becomes the classical Hardy operator

$$
H f(x)=\frac{1}{x} \int_{0}^{x} f(t) d t
$$

and if $\mu=\sum_{k=1}^{\infty} \delta_{k}$, then $H_{\mu}$ becomes the discrete Hardy operator

$$
\mathcal{H} f(k)=\frac{f(1)+\cdots+f(k)}{k} .
$$

For $1<p<\infty$, reference [1] showed that the two operators are bounded on $L^{p}$ and $l^{p}$ respectively. Moreover, for both, the best constants are $p /(p-1)$ and the maximizing functions do not exist. We refer the reader to [2-6] for the background material and further references.

Hardy operator has a close relationship with Hardy-Littlewood maximal operator. From the point of rearrangement, $H f$ is equivalent to $M f$ (see reference [7]). In reference [8], Grafakos considered the $L^{p}$-boundedness for the maximal functions associated with general measures. In this paper, we shall discuss the sharp problems about $H_{\mu}$. We will show that the operator $H_{\mu}$ is bounded on $L^{p}(d \mu)$ with an upper bound no more than

(c) The Author(s) 2018. This article is distributed under the terms of the Creative Commons Attribution 4.0 International License (http://creativecommons.org/licenses/by/4.0/), which permits unrestricted use, distribution, and reproduction in any medium, provided you give appropriate credit to the original author(s) and the source, provide a link to the Creative Commons license, and indicate if changes were made. 
$p /(p-1)$. Furthermore, we will characterize a sufficient condition about $\mu$ such that $\left\|H_{\mu}\right\|_{L^{p} \rightarrow L^{p}}=p /(p-1)$.

From the definition about $H_{\mu}$, it is not necessary to consider the points $x$ such that $\mu([0, x])=0$ or $\infty$. Therefore, we let

$$
a=\inf \{x: \mu([0, x])>0\},
$$

and

$$
b= \begin{cases}\infty & \text { if } B=\emptyset, \\ \inf B & \text { if } B \neq \emptyset\end{cases}
$$

where $B$ denotes the set $\{x: \mu([0, x])=\infty$ or $\mu([x, \infty))=0\}$. Then we call that the measure $\mu$ is supported in the interval $[a, b]$.

For the case of weak type inequality, the best constant from $L^{p}(d \mu)$ to $L^{p, \infty}(d \mu)$ is always 1 .

Theorem 1.1 Let $\mu$ be a positive measure on $[0, \infty]$ and $1 \leq p<\infty$. Then we have

$$
\left\|H_{\mu}\right\|_{L^{p}(d \mu) \rightarrow L^{p, \infty}(d \mu)}=1 .
$$

Theorem 1.2 Suppose that $\mu$ is supported in $[a, b]$ and $f \in L^{p}(d \mu)$ with $1<p<\infty$. For $f \neq 0$, define

$$
\mathcal{R}_{\mu}(f)=\frac{\left\|H_{\mu} f\right\|_{L^{p}(d \mu)}}{\|f\|_{L^{p}(d \mu)}}
$$

Then the following statements hold:

(i) $\left\|H_{\mu} f\right\|_{L^{p}(d \mu)} \leq \frac{p}{p-1}\|f\|_{L^{p}(d \mu)}$ holds for arbitrary positive measure $\mu$.

(ii) There exists no function $f$ such that $\mathcal{R}_{\mu}(f)=\frac{p}{p-1}$ holds.

Theorem 1.3 If $\mu$ satisfies one of the following conditions:

Condition 1. $\mu([a, b])=\infty$ and

$$
\lim _{x \rightarrow b} \frac{\mu([a, x])}{\mu([a, x))}=1
$$

Condition 2. $\{a\}$ is not an atom of $\mu$, and

$$
\lim _{x \rightarrow a} \frac{\mu([a, x])}{\mu([a, x))}=1
$$

then we have

$$
\sup _{f \in L^{p}(d \mu), f \neq 0} \mathcal{R}_{\mu}(f)=\frac{p}{p-1} .
$$

We remark that there indeed exist some measures so that

$$
\sup _{f \in L^{p}(d \mu), f \neq 0} \mathcal{R}_{\mu}(f)<\frac{p}{p-1} .
$$


For example, it is easy to know that the Dirac measure $\delta_{0}$ satisfies inequality (3). In this paper, we will give some more complex counterexamples.

\section{Preliminary and lemmas}

In the study of sharp problems, the rearrangement of function is a very useful tool. Let

$$
d_{f}(s)=\mu(\{|f|>s\}) .
$$

Then the rearrangement of $f$ is defined by

$$
f^{*}(t)=\inf \left\{s>0: d_{f}(s) \leq t\right\}
$$

By the properties of the rearrangement, we can easily have

$$
\|f\|_{L^{p}(d \mu)}=\left\|f^{*}\right\|_{L^{p}(d m)} .
$$

We refer the reader to [9] for more properties of rearrangement. In reference [1], Hardy gave the following result.

Lemma 2.1 (G.H. Hardy and J.E. Littlewood) Let $(X, \mu)$ be a measurable space. Iff, $g \in$ $\mathcal{M}(X, \mu)$, then

$$
\int_{X}|f g| d \mu \leq \int_{0}^{\infty} f^{*}(t) g^{*}(t) d t
$$

holds.

Moreover, the theory of rearrangement plays an important role in proving the existence of maximizing function. This is because of the following lemma introduced by Lieb [10].

Lemma 2.2 Suppose that $(M, \Sigma, \mu)$ and $\left(M^{\prime}, \Sigma^{\prime}, \mu^{\prime}\right)$ are two measure spaces. Let $X$ and $Y$ be $L^{p}(M, \Sigma, \mu)$ and $L^{q}\left(M^{\prime}, \Sigma^{\prime}, \mu^{\prime}\right)$ with $1 \leq p \leq q<\infty$. Let $A$ be a bounded linear operator from $X$ to $Y$. For $f \in X$ with $f \neq 0$, set

$$
\mathcal{R}(f)=\frac{\|A f\|_{Y}}{\|f\|_{X}}
$$

and

$$
N=\sup \{\mathcal{R}(f): f \neq 0\}
$$

Let $\left\{f_{j}\right\}$ be a uniform norm-bounded maximizing sequence for $N$, and assume that $f_{j} \rightarrow f \neq 0$ and that $A\left(f_{j}\right) \rightarrow A(f)$ pointwise almost everywhere. Then $f$ maximizes, i.e., $\mathcal{R}(f)=N$.

\section{The boundedness of weak- $L^{p}$}

In this section, we first prove Theorem 1.1. For the sake of clarity, we define a function as

$$
F_{\mu}(x):=\mu([0, x])
$$


Obviously $F_{\mu}$ increases as $x \rightarrow \infty$. It follows from Lemma 2.1 and the definition of $H_{\mu}$ that

$$
\begin{aligned}
H_{\mu} f(x) & =\frac{1}{\mu([0, x])} \int_{[0, x]} f(t) d \mu(t) \\
& \leq \frac{1}{F_{\mu}(x)} \int_{\left[0, F_{\mu}(x)\right]} f^{*}(t) d t \\
& =H f^{*}\left(F_{\mu}(x)\right) .
\end{aligned}
$$

Let

$$
E_{\mu}^{f^{*}}(\lambda):=\left\{x: H f^{*}\left(F_{\mu}(x)\right)>\lambda\right\}
$$

Note that $f^{*}$ decreases, so we easily have that $H f^{*}$ decreases as well. If we take

$$
x_{0}=\sup \left\{x: H f^{*}\left(F_{\mu}(x)\right)>\lambda\right\}
$$

then it implies that

$$
E_{\mu}^{f^{*}}(\lambda)=\left[0, x_{0}\right)
$$

Thus, we can obtain that

$$
\left\{x: H f^{*}(x)>\lambda\right\} \supset\left[0, F_{\mu}\left(x_{0}\right)\right) .
$$

We conclude that

$$
\mu\left(\left\{x: H f^{*}\left(F_{\mu}(x)\right)>\lambda\right\}\right) \leq F_{\mu}\left(x_{0}\right) \leq\left|\left\{x: H f^{*}(x)>\lambda\right\}\right|,
$$

where $|\cdot|$ denotes the Lebesgue measure. It follows from inequalities (4) and (5) that

$$
\begin{aligned}
\frac{\sup _{\lambda>0} \lambda \mu\left(\left\{x: H_{\mu} f(x)>\lambda\right\}\right)^{\frac{1}{p}}}{\|f\|_{L^{p}(d \mu)}} & \leq \frac{\sup _{\lambda>0} \lambda \mu\left(\left\{x: H f^{*}\left(F_{\mu}(x)\right)>\lambda\right\}\right)^{\frac{1}{p}}}{\left\|f^{*}\right\|_{L^{p}(d m)}} \\
& \leq \frac{\sup _{\lambda>0} \lambda\left|\left\{x: H f^{*}(x)>\lambda\right\}\right|^{\frac{1}{p}}}{\left\|f^{*}\right\|_{L^{p}(d m)}} .
\end{aligned}
$$

Since $f^{*} \in L^{p}(d m)$, by Hölder's inequality, we have that

$$
H f^{*}(x)=\frac{1}{x} \int_{0}^{x} f^{*}(t) d t \leq\left(\frac{1}{x} \int_{0}^{x}\left|f^{*}(t)\right|^{p} d t\right)^{\frac{1}{p}} \leq x^{-\frac{1}{p}}\left\|f^{*}\right\|_{L^{p}(d m)} .
$$

Thus it is obvious to obtain that

$$
\left|\left\{x: H f^{*}(x)>\lambda\right\}\right| \leq\left|\left\{x: x^{-\frac{1}{p}}\left\|f^{*}\right\|_{L^{p}(d m)}>\lambda\right\}\right|=\frac{\left\|f^{*}\right\|_{L^{p}(d m)}^{p}}{\lambda^{p}} .
$$

From inequality (6) and inequality (8), we have

$$
\frac{\sup _{\lambda>0} \lambda \mu\left(\left\{x: H_{\mu} f(x)>\lambda\right\}\right)^{\frac{1}{p}}}{\|f\|_{L^{p}(d \mu)}} \leq 1 .
$$


That is,

$$
\frac{\left\|H_{\mu} f\right\|_{L^{p, \infty}(d \mu)}}{\|f\|_{L^{p}(d \mu)}} \leq 1
$$

holds. This is equivalent to

$$
\left\|H_{\mu}\right\|_{L^{p}(d \mu) \rightarrow L^{p, \infty}(d \mu)} \leq 1
$$

Next it suffices to show that the constant 1 is sharp for inequality (10).

Take $0 \leq x_{1}<x_{2}<\infty$ such that $0<\mu\left(\left[x_{1}, x_{2}\right]\right)<\infty$. Let $g=\chi_{\left[x_{1}, x_{2}\right]}$. It is easy to obtain

$$
\left\|H_{\mu} g\right\|_{L^{p, \infty}(d \mu)}=\|g\|_{L^{p}(d \mu)} .
$$

The proof is completed.

\section{$4 L^{p}$-boundedness of the operator $H_{\mu}$ with upper bound $p /(p-1)$}

Now we will show the results (i) and (ii) of Theorem 1.2.

Proof Following the proof of (5), we obtain

$$
\int_{[0, \infty]}(f(\mu([0, x])))^{p} d \mu(x) \leq \int_{[0, \infty]} f^{p}(x) d x
$$

By inequality (11), we conclude that

$$
\begin{aligned}
\left\|H_{\mu} f\right\|_{L^{p}\left(\mathbb{R}_{+}, d \mu\right)} & =\left(\int_{\mathbb{R}_{+}}\left|\frac{1}{\mu([0, x])} \int_{[0, x]} f(t) d \mu(t)\right|^{p} d \mu(x)\right)^{\frac{1}{p}} \\
& \leq\left(\int_{\mathbb{R}_{+}}\left|\frac{1}{F_{\mu}(x)} \int_{\left[0, F_{\mu}(x)\right]} f^{*}(t) d t\right|^{p} d \mu(x)\right)^{\frac{1}{p}} \\
& =\left(\int_{\mathbb{R}_{+}}\left|H f^{*}\left(F_{\mu}(x)\right)\right|^{p} d \mu(x)\right)^{\frac{1}{p}} \\
& \leq\left(\int_{\mathbb{R}_{+}}\left|H f^{*}(x)\right|^{p} d x\right)^{\frac{1}{p}} .
\end{aligned}
$$

It follows from the inequality of classical Hardy operator that

$$
\left(\int_{\mathbb{R}_{+}}\left|H f^{*}(x)\right|^{p} d x\right)^{\frac{1}{p}} \leq \frac{p}{p-1}\left\|f^{*}\right\|_{L^{p}(d m)}=\frac{p}{p-1}\|f\|_{L^{p}(d \mu)} .
$$

Combining inequality (12) with inequality (13), we have

$$
\left\|H_{\mu} f\right\|_{L^{p}\left(\mathbb{R}_{+}, d \mu\right)} \leq \frac{p}{p-1}\|f\|_{L^{p}(d \mu)}
$$

Since the sharp function for the classical Hardy operator does not exist, it is easy to know from inequality (12) that there exists no function $f$ such that $\mathcal{R}_{\mu}(f)=\frac{p}{p-1}$. The proof of the result (ii) of Theorem 1.2 is completed. 
5 A characterization of the measure $\mu$ which ensures $\sup _{f \neq 0} \mathcal{R}_{\mu}(f)=p /(p-1)$

In this section, we try to characterize the measure $\mu$ which ensures $\sup _{f \neq 0} \mathcal{R}_{\mu}(f)=p /(p-$ 1 ). We regard $\mu$ as a complete atom measure by giving an appropriate partition on $[0, \infty]$. We first present a partition on $[0, \infty]$ by the following two lemmas.

Lemma 5.1 Let $\mu$ be a positive measure that is supported on $[0, \infty]$. If $\mu([0, \infty])=\infty$ and

$$
\lim _{x \rightarrow \infty} \frac{\mu(\{x\})}{\mu([0, x])}=0
$$

then there exists a partition on $[0, \infty]$ as

$$
I_{0}=\left[0, x_{1}\right], \quad I_{1}=\left(x_{1}, x_{2}\right], \ldots, \quad I_{k}=\left(x_{k}, x_{k+1}\right], \ldots
$$

such that

$$
\mu\left(I_{k+1}\right) \geq \mu\left(I_{k}\right)
$$

and

$$
\lim _{k \rightarrow \infty} \frac{\mu\left(I_{k}\right)}{\mu\left(\left[0, x_{k+1}\right]\right)}=0
$$

Proof Let $x_{1}$ be any positive number. Denote $I_{0}=\left[0, x_{1}\right]$. Since $\mu$ is supported on $[0, \infty]$, we have

$$
\mu\left(I_{0}\right)>0
$$

For $k=2$, we let

$$
x_{2}=\inf \left\{x: \mu\left(\left(x_{1}, x\right]\right) \geq \mu\left(\left[0, x_{1}\right]\right)\right\} .
$$

For $k>2$, we let

$$
x_{k}=\inf \left\{x: \mu\left(\left(x_{k-1}, x\right]\right) \geq \mu\left(\left(x_{k-2}, x_{k-1}\right]\right)\right\} .
$$

Denote $I_{k}=\left[x_{k-1}, x_{k}\right]$ with $k=2,3, \ldots$. Since $\mu([0, \infty])=\infty$, we easily have

$$
\lim _{k \rightarrow \infty} x_{k}=\infty
$$

Thus, $\left\{I_{k}\right\}$ obviously constitutes a partition of $[0, \infty]$.

We first show that

$$
\mu\left(I_{k}\right) \geq \mu\left(I_{k-1}\right)
$$

and

$$
\mu\left(\left(x_{k}, x_{k+1}\right)\right) \leq \mu\left(I_{k-1}\right) .
$$


By our construction, for any $x>x_{k+1}$, it follows that

$$
\mu\left(\left(x_{k}, x\right]\right) \geq \mu\left(\left(I_{k-1}\right)\right) .
$$

Thus the property of measure implies that

$$
\mu\left(I_{k}\right)=\lim _{\substack{x>x_{k+1} \\ x \rightarrow x_{k+1}}} \mu\left(\left(x_{k}, x\right]\right) \geq \mu\left(I_{k-1}\right) .
$$

Moreover, if $x_{k}<x<x_{k+1}$, then $\mu\left(\left[x_{k}, x\right]\right)<\mu\left(I_{k-1}\right)$. Thus, it follows that

$$
\mu\left(\left(x_{k}, x_{k+1}\right)\right)=\lim _{\substack{x<x_{k+1} \\ x \rightarrow x_{k+1}}} \mu\left(\left(x_{k}, x\right]\right) \leq \mu\left(I_{k-1}\right) .
$$

To complete the proof, it remains to show that

$$
\lim _{k \rightarrow \infty} \frac{\mu\left(I_{k-1}\right)}{\mu\left(\left[0, x_{k}\right]\right)}=0 .
$$

This is equivalent to prove that, for any $\epsilon>0$, there is an integer $N>0$ such that

$$
\frac{\mu\left(I_{k-1}\right)}{\mu\left(\left[0, x_{k}\right]\right)} \leq 2 \epsilon
$$

holds for $k \geq N$.

In order to prove this result, we divide the set $\mathbb{Z}^{+} \backslash\{1\}$ into two parts:

$$
F_{\epsilon}:=\left\{k \in \mathbb{Z}: k \geq 2, \frac{\mu\left(\left\{x_{k}\right\}\right)}{\mu\left(\left(x_{k-1}, x_{k}\right)\right)}<\epsilon\right\}
$$

and

$$
G_{\epsilon}:=\left\{k \in \mathbb{Z}: k \geq 2, \frac{\mu\left(\left\{x_{k}\right\}\right)}{\mu\left(\left(x_{k-1}, x_{k}\right)\right)} \geq \epsilon\right\} .
$$

By definition (16), if $k \in G_{\epsilon}$, then we have

$$
\mu\left(I_{k-1}\right) \leq\left(1+\frac{1}{\epsilon}\right) \mu\left(\left\{x_{k}\right\}\right)
$$

We discuss the problem in two cases:

Case I. $G_{\epsilon}$ is not a finite set.

Case II. $G_{\epsilon}$ is a finite set.

If $G_{\epsilon}$ is not a finite set, then by equality $\lim _{x \rightarrow \infty} \frac{\mu(\{x\})}{\mu([0, x])}=0$, there exists an integer $N \in G_{\epsilon}$ such that, for any $k \geq N$,

$$
\frac{\mu\left(\left\{x_{k}\right\}\right)}{\mu\left(\left[0, x_{k}\right]\right)}<\frac{\epsilon^{2}}{1+\epsilon}
$$


Thus if $k>N$ and $k \in G_{\epsilon}$, then by inequalities (17) and (18), we have

$$
\frac{\mu\left(I_{k-1}\right)}{\mu\left(\left[0, x_{k}\right]\right)} \leq \epsilon
$$

On the other hand, if $k>N$ and $k \in F_{\epsilon}$, since $G_{\epsilon}$ is not a finite integer and $N \in G_{\epsilon}$, we can find a series of integers $k_{0}, k_{0}+1, \ldots, k$, such that $k_{0} \in G_{\epsilon}$, and

$$
k_{0}+1, \ldots, k \in F_{\epsilon} \text {. }
$$

By the definition of $F_{\epsilon}$ and inequality (14), we can conclude that if $i \in F_{\epsilon}$, then

$$
\begin{aligned}
\mu\left(\left(x_{i-1}, x_{i}\right]\right) & =\mu\left(\left(x_{i-1}, x_{i}\right)\right)+\mu\left(\left\{x_{i}\right\}\right) \\
& \leq(1+\epsilon) \mu\left(\left(x_{i-1}, x_{i}\right)\right) \\
& \leq(1+\epsilon) \mu\left(\left(x_{i-2}, x_{i-1}\right]\right) .
\end{aligned}
$$

It immediately implies from inequality (20) that

$$
\begin{aligned}
\mu\left(\left(x_{k_{0}}, x_{k}\right]\right) & =\sum_{i=k_{0}+1}^{k} \mu\left(\left(x_{i-1}, x_{i}\right]\right) \\
& \geq \sum_{i=k_{0}+1}^{k}(1+\epsilon)^{i-k} \mu\left(\left(x_{k-1}, x_{k}\right]\right) \\
& =\mu\left(\left(x_{k-1}, x_{k}\right]\right) \frac{1-\left(\frac{1}{1+\epsilon}\right)^{k-k_{0}}}{1-\frac{1}{1+\epsilon}} .
\end{aligned}
$$

Thus, by inequality (21), we have

$$
\begin{aligned}
\frac{\mu\left(I_{k-1}\right)}{\mu\left(\left[0, x_{k}\right]\right)} & \leq \frac{\mu\left(\left(x_{k-1}, x_{k}\right]\right)}{\mu\left(\left(x_{k_{0}-1}, x_{k}\right]\right)} \leq \frac{1-\frac{1}{1+\epsilon}}{1-\left(\frac{1}{1+\epsilon}\right)^{k-k_{0}}} \\
& \leq \frac{\epsilon}{1-\left(\frac{1}{1+\epsilon}\right)^{k-k_{0}}} .
\end{aligned}
$$

Since $k_{0} \in G_{\epsilon}$, inequalities (14) and (20) imply

$$
\frac{\mu\left(I_{k-1}\right)}{\mu\left(\left[0, x_{k}\right]\right)} \leq(1+\epsilon)^{k-k_{0}} \frac{\mu\left(I_{k_{0}-1}\right)}{\mu\left(\left[0, x_{k}\right]\right)} \leq(1+\epsilon)^{k-k_{0}} \epsilon .
$$

If $(1+\epsilon)^{k-k_{0}}>2$, by inequality (22), we have

$$
\frac{\mu\left(I_{k-1}\right)}{\mu\left(\left[0, x_{k}\right]\right)} \leq 2 \epsilon
$$

If $(1+\epsilon)^{k-k_{0}} \leq 2$, by inequality (23), we have

$$
\frac{\mu\left(I_{k-1}\right)}{\mu\left(\left[0, x_{k}\right]\right)} \leq 2 \epsilon
$$


At last, we conclude that if $k>N$ and $k \in F_{\epsilon}$, then

$$
\frac{\mu\left(I_{k-1}\right)}{\mu\left(\left[0, x_{k}\right]\right)} \leq 2 \epsilon
$$

The proof of Case I is complete.

If $G_{\epsilon}$ is a finite set, then we can find an integer $k_{0}$ such that $k \in F_{\epsilon}$ for $k>k_{0}$. Then, by inequality (22), we can find a big enough integer $N$ such that

$$
\frac{\mu\left(I_{k-1}\right)}{\mu\left(\left[0, x_{k}\right]\right)} \leq 2 \epsilon
$$

if $k \geq N$. The proof is completed.

Lemma 5.2 Suppose that $\mu$ is supported in $[0, \infty]$. If $\mu(\{0\})=0$ and $\lim _{x \rightarrow 0} \frac{\mu([0, x])}{\mu([0, x))}=1$, then there exists a partition on $(0,1]$,

$$
\left(x_{1}, 1\right],\left(x_{2}, x_{1}\right], \ldots,\left(x_{k}, x_{k-1}\right], \ldots,
$$

such that $\lim _{k \rightarrow \infty} x_{k}=0$ and

$$
\lim _{k \rightarrow \infty} \frac{\mu\left(\left(x_{k}, x_{k-1}\right]\right)}{\mu\left(\left[0, x_{k-1}\right]\right)}=0
$$

Proof Without loss of generality, suppose

$$
\mu([0,1])=\sum_{k=1}^{\infty} \frac{1}{k^{2}} .
$$

If $\mu(\{1\})<1$, then we set $k_{0}=0$. If $\mu(\{1\}) \geq 1$, then we set

$$
k_{0}=\max \left\{m: \sum_{k=1}^{m} \frac{1}{k^{2}} \leq \mu(\{1\})\right\} .
$$

It is easy to see that

$$
\sum_{k=1}^{k_{0}+1} \frac{1}{k^{2}}>\mu(\{1\}) .
$$

Then we can find a positive real number $x_{1}<1$ such that

$$
x_{1}=\sup \left\{x: \mu((x, 1]) \geq \sum_{k=1}^{k_{0}+1} \frac{1}{k^{2}}\right\} .
$$

Proceeding in this way, we set

$$
k_{i}=\max \left\{m: \sum_{k=1}^{m} \frac{1}{k^{2}} \leq \mu\left(\left[x_{i}, 1\right]\right)\right\}
$$


and

$$
x_{i+1}=\sup \left\{x: \mu((x, 1]) \geq \sum_{k=1}^{k_{i}+1} \frac{1}{k^{2}}\right\}
$$

for $i \geq 1$. By (27), (25), and (26), we can conclude

$$
\sum_{k=1}^{k_{i}} \frac{1}{k^{2}} \leq \mu\left(\left[x_{i}, 1\right]\right) \leq \mu\left(\left(x_{i+1}, 1\right]\right) \leq \sum_{k=1}^{k_{i}+1} \frac{1}{k^{2}} \leq \mu\left(\left[x_{i+1}, 1\right]\right) .
$$

It is easy to see that $x_{i}>x_{i+1}$ and

$$
\lim _{i \rightarrow \infty} \mu\left(\left[x_{i}, 1\right]\right) \geq \lim _{i \rightarrow \infty} \sum_{k=1}^{k_{i}} \frac{1}{k^{2}}=\mu((0,1]) .
$$

Thus we have $\lim _{i \rightarrow \infty} x_{i}=0$. It is easy to see that

$$
\left(x_{1}, 1\right],\left(x_{2}, x_{1}\right], \ldots,\left(x_{k}, x_{k-1}\right], \ldots,
$$

divide $(0,1]$. It can be implied from inequality $(27)$ that

$$
\mu\left(\left[x_{i}, 1\right]\right)+\frac{1}{\left(k_{i}+1\right)^{2}} \geq \sum_{k=1}^{k_{i}+1} \frac{1}{k^{2}} \geq \mu\left(\left(x_{i+1}, 1\right]\right) .
$$

To prove this partition satisfying the requirement of the lemma, we define two integer sets:

$$
F_{\epsilon}=\left\{k \geq 1: \frac{\mu\left(\left\{x_{k}\right\}\right)}{\mu\left(\left(x_{k+1}, x_{k}\right]\right)}<\epsilon\right\}
$$

and

$$
G_{\epsilon}=\left\{k \geq 1: \frac{\mu\left(\left\{x_{k}\right\}\right)}{\mu\left(\left(x_{k+1}, x_{k}\right]\right)} \geq \epsilon\right\},
$$

where $\epsilon$ is an arbitrary positive real number. Since $\lim _{x \rightarrow 0} \frac{\mu([0, x])}{\mu([0, x))}=1$, we have $\lim _{x \rightarrow 0} \frac{\mu(\{x\}))}{\mu([0, x))}=0$. It is easy to find an integer $N$ such that

$$
\frac{\mu\left(\left\{x_{i-1}\right\}\right)}{\mu\left(\left[0, x_{i-1}\right]\right)}<2 \epsilon^{2}
$$

for any integer $i>N$. Thus, by the construction of $G_{\epsilon}$, if $i>N$ and $i \in G_{\epsilon}$, we have

$$
\frac{\mu\left(\left(x_{i}, x_{i-1}\right]\right)}{\mu\left(\left[0, x_{i-1}\right]\right)}<2 \epsilon .
$$

If $i \in F_{\epsilon}$, then we have

$$
\mu\left(\left(x_{i+1}, x_{i}\right]\right) \leq \frac{1}{1-\epsilon} \mu\left(\left(x_{i+1}, x_{i}\right)\right) .
$$


By inequalities (28) and (29), we have

$$
\begin{aligned}
\frac{\mu\left(\left(x_{i+1}, x_{i}\right]\right)}{\mu\left(\left[0, x_{i}\right]\right)} & \leq \frac{1}{1-\epsilon} \frac{\mu\left(\left(x_{i+1}, x_{i}\right)\right)}{\mu\left(\left[0, x_{i}\right]\right)} \\
& =\frac{1}{1-\epsilon} \frac{\mu\left(\left(x_{i+1}, 1\right]\right)-\mu\left(\left[x_{i}, 1\right]\right)}{\mu((0,1])-\mu\left(\left(x_{i}, 1\right]\right)} \\
& \leq \frac{1}{1-\epsilon} \frac{1 /\left(k_{i}+1\right)^{2}}{\sum_{k=k_{i}+2}^{\infty} 1 / k^{2}} .
\end{aligned}
$$

Thus we can find a sufficiently large integer which is still denoted by $N$ such that, for any integer $i>N$ and $i \in F_{\epsilon}$, there is

$$
\frac{\mu\left(\left(x_{i}, x_{i-1}\right]\right)}{\mu\left(\left[0, x_{i-1}\right]\right)}<2 \epsilon .
$$

Since $\epsilon$ is an arbitrary real number, we have

$$
\lim _{k \rightarrow \infty} \frac{\mu\left(\left(x_{k}, x_{k-1}\right]\right)}{\mu\left(\left[0, x_{k-1}\right]\right)}=0 .
$$

The proof is completed.

After finishing our preparations, we can give the proof of the result (iii) of the main theorem.

Proof Let

$$
T_{a, b}(x)= \begin{cases}\tan \left(\frac{\pi}{2}\left(\frac{x-a}{b-a}\right)\right), & 0<b<\infty ; \\ x-a, & b=\infty .\end{cases}
$$

By equality (30), we can obtain a new measure denoted by $\mu_{T}$ which is supported in $[0, \infty]$ so that, for any open interval $(x, y)$, we have

$$
\mu_{T}((x, y))=\mu\left(\left(T_{a, b}^{-1}(x), T_{a, b}^{-1}(y)\right)\right) .
$$

Then it is easy to get

$$
\sup \left\{\mathcal{R}_{\mu} f \mid f \in L^{p}(d \mu)\right\}=\sup \left\{\mathcal{R}_{\mu_{T}} f \mid f \in L^{p}\left(d \mu_{T}\right)\right\} .
$$

Thus it is enough to assume that the measure $\mu$ is supported in $[0, \infty]$.

We first consider Condition 1.

By Lemma 5.1, we can divide $\mathbb{R}^{+}$into a series of intervals

$$
\left[0, x_{1}\right],\left(x_{1}, x_{2}\right], \ldots,\left(x_{k}, x_{k+1}\right], \ldots,
$$

such that

$$
\lim _{k \rightarrow \infty} \frac{\mu\left(\left(x_{k}, x_{k+1}\right]\right)}{\mu\left(\left[0, x_{k}\right]\right)}=0 .
$$


For any $\epsilon>0$, if we can find a function $f_{\epsilon}$ such that $\mathcal{R}\left(f_{\epsilon}\right) \geq \frac{p}{p-1}-O(\epsilon)$, then the proof is completed.

By the property of the partition, there exists an integer $N$ satisfying

$$
\frac{\mu\left(\left(x_{k}, x_{k+1}\right]\right)}{\mu\left(\left[0, x_{k}\right]\right)}<\epsilon
$$

for $k \geq N$. This inequality is equivalent to

$$
\frac{\mu\left(\left[0, x_{k+1}\right]\right)}{\mu\left(\left[0, x_{k}\right]\right)}<1+\epsilon
$$

Let

$$
f_{\epsilon}=\sum_{k=N}^{\infty} \mu\left(\left[0, x_{k+1}\right]\right)^{-\frac{1}{p}-\epsilon} \chi_{\left(x_{k}, x_{k+1}\right]} .
$$

First we estimate the norm of $f_{\epsilon}$

$$
\begin{aligned}
\left\|f_{\epsilon}\right\|_{L^{p}(d \mu)} & =\left(\sum_{k=N}^{\infty} \mu\left(\left[0, x_{k+1}\right]\right)^{-1-p \epsilon} \mu\left(\left(x_{k}, x_{k+1}\right]\right)\right)^{\frac{1}{p}} \\
& \geq\left(\frac{1}{1+\epsilon}\right)^{\frac{1}{p}+\epsilon}\left(\sum_{k=N}^{\infty} \mu\left(\left[0, x_{k}\right]\right)^{-1-p \epsilon} \mu\left(\left(x_{k}, x_{k+1}\right]\right)\right)^{\frac{1}{p}} \\
& =\left(\frac{1}{1+\epsilon}\right)^{\frac{1}{p}+\epsilon}\left(\sum_{k=N}^{\infty} \int_{\mu\left(\left[0, x_{k}\right]\right)}^{\mu\left(\left[0, x_{k+1}\right]\right)} \mu\left(\left[0, x_{k}\right]\right)^{-1-p \epsilon} d t\right)^{\frac{1}{p}} \\
& \geq\left(\frac{1}{1+\epsilon}\right)^{\frac{1}{p}+\epsilon}\left(\int_{\mu\left(\left[0, x_{N}\right]\right)}^{\infty} t^{-1-p \epsilon} d t\right)^{\frac{1}{p}} \\
& \geq\left(\frac{1}{1+\epsilon}\right)^{\frac{1}{p}+\epsilon}\left(\frac{1}{p \epsilon}\right)^{\frac{1}{p}} \mu\left(\left[0, x_{N}\right]\right)^{-\epsilon} .
\end{aligned}
$$

Next, we estimate the value of $H_{\mu} f_{\epsilon}(x)$. When $k \geq N$ and $x_{k}<x \leq x_{k+1}$, we have

$$
\begin{aligned}
H_{\mu} f_{\epsilon}(x) & =\frac{1}{\mu([0, x])} \int_{[0, x]} f_{\epsilon}(t) d \mu(t) \\
& \geq \frac{1}{\mu\left(\left[0, x_{k+1}\right]\right)} \int_{\left[0, x_{k}\right]} f_{\epsilon}(t) d \mu(t) \\
& =\frac{1}{\mu\left(\left[0, x_{k+1}\right]\right)} \sum_{i=N}^{k-1} \mu\left(\left[0, x_{i+1}\right]\right)^{-\frac{1}{p}-\epsilon} \mu\left(\left(x_{i}, x_{i+1}\right]\right) \\
& \geq\left(\frac{1}{1+\epsilon}\right)^{\frac{1}{p}+\epsilon} \frac{1}{\mu\left(\left[0, x_{k+1}\right]\right)} \sum_{i=N}^{k-1} \mu\left(\left[0, x_{i}\right]\right)^{-\frac{1}{p}-\epsilon} \mu\left(\left(x_{i}, x_{i+1}\right]\right) \\
& =\left(\frac{1}{1+\epsilon}\right)^{\frac{1}{p}+\epsilon} \frac{1}{\mu\left(\left[0, x_{k+1}\right]\right)} \sum_{i=N}^{k-1} \int_{\mu\left(\left[0, x_{i}\right]\right)}^{\mu\left(\left[0, x_{i+1}\right]\right)} \mu\left(\left[0, x_{i}\right]\right)^{-\frac{1}{p}-\epsilon} d t
\end{aligned}
$$




$$
\begin{aligned}
& \geq\left(\frac{1}{1+\epsilon}\right)^{1+\frac{1}{p}+\epsilon} \frac{1}{\mu\left(\left[0, x_{k}\right]\right)} \int_{\mu\left(\left[0, x_{N}\right]\right)}^{\mu\left(\left[0, x_{k}\right]\right)} t^{-\frac{1}{p}-\epsilon} d t \\
& \geq\left(\frac{1}{1+\epsilon}\right)^{1+\frac{1}{p}+\epsilon} \frac{1}{1-\frac{1}{p}-\epsilon}\left(\mu\left(\left[0, x_{k}\right]\right)^{-\frac{1}{p}-\epsilon}-\frac{\mu\left(\left[0, x_{N}\right]\right)^{1-\frac{1}{p}-\epsilon}}{\mu\left(\left[0, x_{k}\right]\right)}\right) .
\end{aligned}
$$

Set

$$
\begin{aligned}
f_{\epsilon}^{(1)} & =\left(\frac{1}{1+\epsilon}\right)^{1+\frac{1}{p}+\epsilon} \frac{1}{1-\frac{1}{p}-\epsilon} \sum_{k=N}^{\infty} \mu\left(\left[0, x_{k}\right]\right)^{-\frac{1}{p}-\epsilon} \chi_{\left(x_{k}, x_{k+1}\right]} \\
& =\left(\frac{1}{1+\epsilon}\right)^{1+\frac{1}{p}+\epsilon} \frac{1}{1-\frac{1}{p}-\epsilon} f_{\epsilon}
\end{aligned}
$$

and

$$
f_{\epsilon}^{(2)}=\left(\frac{1}{1+\epsilon}\right)^{1+\frac{1}{p}+\epsilon} \frac{1}{1-\frac{1}{p}-\epsilon} \sum_{k=N}^{\infty} \frac{\mu\left(\left[0, x_{N}\right]\right)^{1-\frac{1}{p}-\epsilon}}{\mu\left(\left[0, x_{k}\right]\right)} \chi_{\left(x_{k}, x_{k+1}\right]} .
$$

Then we have

$$
\begin{aligned}
\left\|f_{\epsilon}^{(2)}\right\|_{L^{p}(d \mu)} & =\left(\frac{1}{1+\epsilon}\right)^{1+\frac{1}{p}+\epsilon} \frac{1}{1-\frac{1}{p}-\epsilon} \mu\left(\left[0, x_{N}\right]\right)^{1-\frac{1}{p}-\epsilon}\left(\sum_{k=N}^{\infty} \mu\left(\left[0, x_{k}\right]\right)^{-p} \mu\left(\left(x_{k}, x_{k+1}\right]\right)\right)^{\frac{1}{p}} \\
& \leq\left(\frac{1}{1+\epsilon}\right)^{\frac{1}{p}+\epsilon} \frac{1}{1-\frac{1}{p}-\epsilon} \mu\left(\left[0, x_{N}\right]\right)^{1-\frac{1}{p}-\epsilon}\left(\sum_{k=N}^{\infty} \mu\left(\left[0, x_{k+1}\right]\right)^{-p} \mu\left(\left(x_{k}, x_{k+1}\right]\right)\right)^{\frac{1}{p}} \\
& \leq\left(\frac{1}{1+\epsilon}\right)^{\frac{1}{p}+\epsilon} \frac{1}{1-\frac{1}{p}-\epsilon}\left(\frac{1}{p-1}\right)^{\frac{1}{p}} \mu\left(\left[0, x_{N}\right]\right)^{-\epsilon}
\end{aligned}
$$

By inequality (33), we have

$$
\left\|H_{\mu} f_{\epsilon}\right\|_{L^{p}(d \mu)} \geq\left\|f_{\epsilon}^{(1)}\right\|_{L^{p}(d \mu)}-\left\|f_{\epsilon}^{(2)}\right\|_{L^{p}(d \mu)} .
$$

From this result and inequalities (32) and (34), we can get

$$
\begin{aligned}
\mathcal{R}\left(f_{\epsilon}\right) & \geq \frac{\left\|f_{\epsilon}^{(1)}\right\|_{L^{p}(d \mu)}-\left\|f_{\epsilon}^{(2)}\right\|_{L^{p}(d \mu)}}{\left\|f_{\epsilon}\right\|_{L^{p}(d \mu)}} \\
& =\left(\frac{1}{1+\epsilon}\right)^{1+\frac{1}{p}+\epsilon} \frac{1}{1-\frac{1}{p}-\epsilon}-\frac{\left\|f_{\epsilon}^{(2)}\right\|_{L^{p}(d \mu)}}{\left\|f_{\epsilon}\right\|_{L^{p}(d \mu)}} \\
& \geq\left(\frac{1}{1+\epsilon}\right)^{1+\frac{1}{p}+\epsilon} \frac{1}{1-\frac{1}{p}-\epsilon}-\frac{\left(\frac{1}{1+\epsilon}\right)^{\frac{1}{p}+\epsilon} \frac{1}{1-\frac{1}{p}-\epsilon}\left(\frac{1}{p-1}\right)^{\frac{1}{p}}}{\left(\frac{1}{1+\epsilon}\right)^{\frac{1}{p}+\epsilon}\left(\frac{1}{p \epsilon}\right)^{\frac{1}{p}}} .
\end{aligned}
$$

Since $\epsilon$ is arbitrary, it is easy to imply $\sup _{f \neq 0} \mathcal{R}(f)=\frac{p}{p-1}$.

To prove condition (ii), by Lemma 5.2 , we can part the intervals $(0,1]$ to

$$
\left(x_{1}, 1\right],\left(x_{2}, x_{1}\right], \ldots,\left(x_{k+1}, x_{k}\right], \ldots
$$


such that

$$
\lim _{k \rightarrow \infty} \frac{\mu\left(\left(x_{k+1}, x_{k}\right]\right)}{\mu\left(\left(0, x_{k}\right]\right)}=0 .
$$

Then, for any $\epsilon>0$, there is a sufficiently large integer $N$ such that

$$
\frac{\mu\left(\left(x_{k+1}, x_{k}\right]\right)}{\mu\left(\left(0, x_{k}\right]\right)}<\epsilon
$$

for $k \geq N$.

Thus, we have

$$
\frac{\mu\left(\left(0, x_{k+1}\right]\right)}{\mu\left(\left(0, x_{k}\right]\right)} \geq 1-\epsilon
$$

Let $f_{\epsilon}=\sum_{k=N}^{\infty} \mu\left(\left(0, x_{k}\right]\right)^{-\frac{1}{p}+\epsilon} \chi_{\left(x_{k+1}, x_{k}\right]}$. Then, for $x_{k+1}<x \leq x_{k}$ and $k \geq N$, we have

$$
\begin{aligned}
H_{\mu} f_{\epsilon}(x) & =\frac{1}{\mu((0, x])} \int_{(0, x]} f_{\epsilon}(t) d \mu(t) \\
& \geq \frac{1}{\mu\left(\left(0, x_{k}\right]\right)} \int_{\left(0, x_{k+1}\right]} f_{\epsilon}(t) d \mu(t) \\
& =\frac{1}{\mu\left(\left(0, x_{k}\right]\right)} \sum_{i=k+1}^{\infty} \mu\left(\left(0, x_{i}\right]\right)^{-\frac{1}{p}+\epsilon} \mu\left(\left(x_{i+1}, x_{i}\right]\right) \\
& \geq \frac{1}{\mu\left(\left(0, x_{k}\right]\right)} \frac{\mu\left(\left(0, x_{k+1}\right]\right)^{1-\frac{1}{p}+\epsilon}}{1-\frac{1}{p}+\epsilon} \\
& \geq \frac{(1-\epsilon)^{1-\frac{1}{p}+\epsilon}}{1-\frac{1}{p}+\epsilon} \mu\left(\left(0, x_{k}\right]\right)^{-\frac{1}{p}+\epsilon} \\
& =\frac{(1-\epsilon)^{1-\frac{1}{p}+\epsilon}}{1-\frac{1}{p}+\epsilon} f_{\epsilon}(x) .
\end{aligned}
$$

It follows from inequality (36) that

$$
\mathcal{R}\left(f_{\epsilon}\right) \geq \frac{(1-\epsilon)^{1-\frac{1}{p}+\epsilon}}{1-\frac{1}{p}+\epsilon} .
$$

Because $\epsilon$ is arbitrary, it is easy to know $\sup _{f} \mathcal{R}(f) \geq \frac{p}{p-1}$. The proof of the suffice part of Theorem 1.2 is then completed.

\section{Counterexample}

In this section we give some counterexamples that make $\sup _{f \neq 0, f \in L^{p}} \mathcal{R}(f)<p /(p-1)$. The following two lemmas tell us that we can limit our discussion to a special function set.

Lemma 6.1 Suppose $\mu$ is a positive measure on $\mathbb{R}_{+}$and it has an atom $x_{0}$. If $\left\{f_{n}\right\}, n=$ $1,2, \ldots$, is a series of functions satisfying $f_{n}\left(x_{0}\right)=1$ and

$$
\lim _{n \rightarrow \infty} \mathcal{R}_{\mu}\left(f_{n}\right)=\frac{p}{p-1},
$$


then we have

$$
\lim _{n \rightarrow \infty}\left\|f_{n}\right\|_{L^{p}(d \mu)}=\infty
$$

Proof Without loss of generality, we assume that $\mu\left(\left\{x_{0}\right\}\right)=1$. If the assertion does not hold, then we can assume that there exists a constant $C$ satisfying $\left\|f_{n}\right\|_{L^{p}(d \mu)} \leq C$. Let $f_{n}^{*}$ be the decreasing rearrangement of $f_{n}$, then it is easy to get $\left\|f_{n}^{*}\right\|_{L^{p}(d m)} \leq C$ and $f_{n}^{*}(1) \geq 1$. Thus we have $f^{*}(x) \geq 1$ for $0<x \leq 1$. By Helly's theorem, we can assume $\lim _{n \rightarrow \infty} f_{n}^{*}=f^{*}$ almost everywhere. Since $f_{n}^{*}$ is decreasing, we have

$$
C^{p} \geq\left\|f_{n}^{*}\right\|_{L^{p}(d m)}^{p} \geq \int_{[0, R]}\left|f_{n}^{*}(t)\right|^{p} d t \geq R\left|f_{n}^{*}(R)\right|^{p},
$$

which is equivalent to $f_{n}^{*}(R) \leq C R^{-\frac{1}{p}}$. Thus, by the control convergence theorem,

$$
\lim _{n \rightarrow \infty} H f_{n}^{*}(x)=\lim _{n \rightarrow \infty} \frac{1}{x} \int_{[0, x]} f_{n}^{*}(t) d t=H f^{*}(x) .
$$

However, by inequality (12), we have

$$
\mathcal{R}_{m}\left(f_{n}^{*}\right) \geq \mathcal{R}_{\mu}\left(f_{n}\right)
$$

it obviously shows that $\left\{f_{n}^{*}\right\}$ is a maximizing sequence for $H$, i.e.,

$$
\lim _{n \rightarrow \infty} \mathcal{R}\left(f_{n}^{*}\right)=\frac{p}{p-1} .
$$

By $\lim _{n \rightarrow \infty} f_{n}^{*}=f^{*}$ and equality (38), using Lemma 2.2, we get $\mathcal{R}_{m}\left(f^{*}\right)=\frac{p}{p-1}$, which contradicts the result about Hardy operator we have known. The proof is completed.

Lemma 6.2 Suppose $\mu$ is a positive measure on $\mathbb{R}_{+}$and it has an atom $x_{0}$. If

$$
\sup \left\{\mathcal{R}_{\mu} f \mid f \in L^{p}(d \mu)\right\}=\frac{p}{p-1},
$$

then there exists a series of functions $\left\{f_{k}\right\}, k=1,2, \ldots$, and $f_{k}\left(x_{0}\right)=0$ such that

$$
\lim _{k \rightarrow \infty} \mathcal{R}_{\mu}\left(f_{k}\right)=\frac{p}{p-1}
$$

Proof It is obvious that we can assume there exists a series of functions $g_{k}, g_{k}\left(x_{0}\right)=1$, such that

$$
\lim _{k \rightarrow \infty} \mathcal{R}_{\mu}\left(g_{k}\right)=\frac{p}{p-1}
$$

Let

$$
f_{k}(x)= \begin{cases}g_{k}(x), & x \neq x_{0} \\ 0, & x=x_{0}\end{cases}
$$


Then we have

$$
H_{\mu} f_{k}(x)= \begin{cases}H_{\mu} g_{k}(x), & x<x_{0} ; \\ H_{\mu} g_{k}(x)-\mu\left(\left\{x_{0}\right\}\right) / \mu([0, x]), & x \geq x_{0} .\end{cases}
$$

By equality (39), we can get

$$
\left\|H_{\mu}\left(f_{k}\right)\right\|_{L^{p}} \geq\left\|H_{\mu}\left(g_{k}\right)\right\|_{L^{p}}-\left\|\frac{\mu\left(\left\{x_{0}\right\}\right)}{\mu([0, \cdot])} \chi_{\left[x_{0}, \infty\right]}\right\|_{L^{p}} .
$$

On the other hand, it is easy to obtain

$$
\left\|f_{k}\right\|_{L^{p}} \leq\left\|g_{k}\right\|+\mu\left(\left\{x_{0}\right\}\right)^{\frac{1}{p}}
$$

By Lemma 6.1, we know that $\lim _{k \rightarrow \infty}\left\|g_{k}\right\|_{L^{p}(d \mu)}=\infty$ and $\lim _{k \rightarrow \infty} \mathcal{R}_{\mu}\left(g_{k}\right)=p /(p-1)$. By this result, together with inequalities (40) and (41), we can have

$$
\lim _{k \rightarrow \infty} \mathcal{R}_{\mu}\left(f_{k}\right)=\frac{p}{p-1}
$$

Now we can give some counterexamples.

Example 6.3 Suppose that $\mu$ is supported in $[a, b), \mu(\{a\})>0$, and $\mu\left(\mathbb{R}_{+}\right)<\infty$. Then $\sup _{f \neq 0} \mathcal{R}_{\mu}(f)<p /(p-1)$.

Proof Suppose that the result is not valid. By Lemma 6.2, we can find a series of functions $\left\{f_{k}\right\}, f_{k}(a)=0$, such that

$$
\lim _{k \rightarrow \infty} \mathcal{R}_{\mu}\left(f_{k}\right)=\frac{p}{p-1}
$$

Let $A=\mu(\{a\}), B=\mu\left(\mathbb{R}_{+}\right)$, and $\mu_{1}=\mu-A \delta_{a}$. Then we have

$$
H_{\mu} f_{k}(x)=\frac{\mu_{1}([0, x])}{\mu([0, x])} \frac{1}{\mu_{1}([0, x])} \int_{[0, x]} f_{k} d \mu_{1} \leq \frac{B-A}{B} H_{\mu_{1}} f_{k}(x)
$$

and

$$
\left\|f_{k}\right\|_{L^{p}(d \mu)}=\left\|f_{k}\right\|_{L^{p}\left(d \mu_{1}\right)}
$$

By inequalities (42) and (43), we obtain

$$
\mathcal{R}_{\mu}\left(f_{k}\right) \leq \frac{B-A}{B} \mathcal{R}_{\mu_{1}}\left(f_{k}\right) \leq \frac{B-A}{B} \frac{p}{p-1} .
$$

It contradicts with $\lim _{k \rightarrow \infty} \mathcal{R}_{\mu}\left(f_{k}\right)=p /(p-1)$. Then the counterexample is valid.

Example 6.4 If $\mu=\sum_{k=-\infty}^{\infty} \lambda^{k} \delta_{\lambda^{k}}$ with $\lambda>1$, then $\sup _{f \in L^{p}(d \mu)} \mathcal{R} f<\frac{p}{p-1}$. 
Proof By the definition of $\mu$, we have

$$
\begin{aligned}
H_{\mu} f\left(\lambda_{k}\right) & =\frac{1}{\mu\left(\left[0, \lambda_{k}\right]\right)} \int_{\left[0, \lambda_{k}\right]} f(t) d \mu(t) \\
& =\frac{(\lambda-1) \sum_{i=-\infty}^{k} \lambda^{i} f\left(\lambda^{i}\right)}{\lambda^{k+1}} \\
& =\frac{\lambda-1}{\lambda} \sum_{i=-\infty}^{0} \lambda^{i} f\left(\lambda^{i+k}\right)
\end{aligned}
$$

and

$$
\begin{aligned}
\left\|f\left(\lambda^{i} \cdot\right)\right\|_{L^{p}(d \mu)} & =\left(\sum_{k=-\infty}^{\infty}\left|f\left(\lambda^{i+k}\right)\right|^{p} \lambda^{k}\right)^{\frac{1}{p}} \\
& =\left(\sum_{k=-\infty}^{\infty}\left|f\left(\lambda^{k}\right)\right|^{p} \lambda^{k-i}\right)^{\frac{1}{p}} \\
& =\lambda^{-\frac{i}{p}}\|f\|_{L^{p}(d \mu)} .
\end{aligned}
$$

By inequalities (44), (45), and Minkowski's inequality, it follows

$$
\begin{aligned}
\left\|H_{\mu} f\right\|_{L^{p}(d \mu)} & =\left\|\frac{\lambda-1}{\lambda} \sum_{i=-\infty}^{0} \lambda^{i} f\left(\lambda^{i} \cdot\right)\right\|_{L^{p}(d \mu)} \\
& \leq \frac{\lambda-1}{\lambda} \sum_{i=-\infty}^{0} \lambda^{i}\left\|f\left(\lambda^{i} \cdot\right)\right\|_{L^{p}(d \mu)} \\
& =\frac{\lambda-1}{\lambda} \sum_{i=-\infty}^{0} \lambda^{i-\frac{i}{p}}\|f\|_{L^{p}(d \mu)} \\
& =\frac{\lambda-1}{\lambda-\lambda^{\frac{1}{p}}}\|f\|_{L^{p}(d \mu)} .
\end{aligned}
$$

It is easy to get $\frac{\lambda-1}{\lambda-\lambda^{\frac{1}{p}}}<\frac{p}{p-1}$. The proof is completed.

Funding

The research was supported by the National Natural Foundation of China (Grant Nos. 11471039, 11271162).

\section{Competing interests}

The authors declare that they have no competing interests.

Authors' contributions

All authors contributed equally and significantly in writing this paper. All authors read and approved the final manuscript.

Author details

${ }^{1}$ Department of Mathematics and Physics, Shijiazhuang Tiedao University, Shijiazhuang, P.R. China. ${ }^{2}$ School of Mathematical Sciences, University of the Chinese Academy of Sciences, Beijing, P.R. China.

\section{Publisher's Note}

Springer Nature remains neutral with regard to jurisdictional claims in published maps and institutional affiliations.

Received: 25 October 2017 Accepted: 20 March 2018 Published online: 10 April 2018 


\section{References}

1. Hardy, G.H., Littlewood, J.E., Pólya, G.: Inequalities. Cambridge University Press, Cambridge (1952)

2. Komori, Y.: Notes on commutators of Hardy operators. Int. J. Pure Appl. Math. 7, 329-334 (2003)

3. Lu, S., Yan, D., Zhao, F.: Sharp bounds for Hardy type operators on higher-dimensional product spaces. J. Inequal. Appl. 2013, 148 (2013)

4. Muckenhoupt, B.: Hardy's inequality with weights. Stud. Math 44(1), 31-38 (1972)

5. Shunchao, L., Jian, W.: Commutators of Hardy operators. J. Math. Anal. Appl. 274(2), 626-644 (2002)

6. Sinnamon, G.: Operators of Lebesgue Spaces with General Measures. McMaster University, Toronto (1987)

7. Bennett, C., Sharpley, R.C.: Interpolation of Operators, vol. 129. Academic Press, San Diego (1988)

8. Grafakos, L., Kinnunen, J.: Sharp inequalities for maximal functions associated with general measures. Proc. R. Soc Edinb., Sect. A, Math. 128(4), 717-723 (1998)

9. Grafakos, L.: Classical and Modern Fourier Analysis. Prentice Hall, New York (2004)

10. Lieb, E.H.: Sharp constants in the Hardy-Littlewood-Sobolev and related inequalities. Ann. Math. 118, 349-374 (1983)

\section{Submit your manuscript to a SpringerOpen ${ }^{0}$ journal and benefit from:}

- Convenient online submission

Rigorous peer review

Open access: articles freely available online

- High visibility within the field

- Retaining the copyright to your article

Submit your next manuscript at $\boldsymbol{~ s p r i n g e r o p e n . c o m ~}$ 